\title{
Numerical simulations of stochastically excited sound waves in a random medium
}

\author{
M. Selwa ${ }^{1}$, R. Skartlien ${ }^{2}$, and K. Murawski ${ }^{1}$ \\ 1 Institute of Physics, UMCS, ul. Radziszewskiego 10, 20-031 Lublin, Poland \\ 2 Norwegian Defence Research Establishment (FFI), Postboks 25, 2027 Kjeller, Norway
}

Received 5 September 2003 / Accepted 4 March 2004

\begin{abstract}
In turbulent acoustic media such as the solar envelope, both wave sources and the propagation characteristics (background density, refractive index, dissipation, etc.) are stochastic quantities. By means of numerical simulation of the Euler equations, we study two cases in a homogeneous stochastic medium in which the background density fluctuations and wave sources are 1) correlated and 2) uncorrelated. We find that in the uncorrelated case, the coherent (or mean) acoustic field is zero, leaving only an incoherent field. In the correlated case, the coherent field is nonzero, yielding both coherent and incoherent fields. We question the use of mean-field dispersion relations to determine frequency shifts in p-mode and f-mode spectra, since the coherent field can be non-existent or weak relative to the incoherent field. We demonstrate the importance of accounting for a stochastic wave source by showing that the two cases give very different frequency shifts.
\end{abstract}

Key words. waves - Sun: interior - Sun: oscillations - convection - turbulence

\section{Introduction}

Motivated by the origin of solar p-modes (e.g., Gough 1994), we discuss generation and propagation of sound waves in a stochastic medium. Simple models acquire much attention as they allow for separation and quantification of effects on the waves from various stochastic quantities in the medium. Indeed, analytical and numerical studies of sound waves in a space-dependent random mass density field reveals that the waves are accelerated and attenuated (Nocera et al. 2001). On the other hand, a time-dependent random mass density field accelerates and amplifies the sound waves (Murawski et al. 2001).

The above mentioned studies treat sound waves in a random medium without discussing the effect of a random wave source. Random wave generation was discussed recently by Skartlien (2002), who used linear theory to show that the existence of the coherent field (or mean field) requires a correlation between the source and the random background fluctuations. Furthermore, it was shown that the power spectrum can be understood as cross power between multiply scattered wave components, and in terms of correlation functions of the random background fluctuations and of the wave source.

The main purpose of this paper is to verify by numerical simulation that the coherent field exists only when there is a correlation between the source and the background fluctuations. We assume $1 \mathrm{~d}$ geometry for the purpose of clear

Send offprint requests to: M. Selwa,

e-mail: mselwa@tytan.umcs. lublin.pl demonstration, but the essential results are valid in $2 \mathrm{~d}$ and $3 \mathrm{~d}$ as well. The paper is organized as follows. Section 2 presents the numerical model. Section 3 describes ensemble averaging and the coherent field. Section 4 contains results of the numerical simulations. Conclusions are found in Sect. 5.

\section{Numerical model}

We limit our discussion to a magnetic-free one-dimensional fluid. For the current problem, it is convenient to add specified sources to the hydrodynamic equations:

$\varrho_{, t}+(\varrho V)_{, x}=S_{\varrho}$,

$(\varrho V)_{, t}+\left(\varrho V^{2}+p\right)_{, x}=V S_{\varrho}+\varrho S_{V}$,

$E_{, t}+[V(E+p)]_{, x}=\frac{1}{2} V^{2} S_{\varrho}+\varrho V S_{V}$.

Here

$E=\frac{p}{\gamma-1}+\frac{1}{2} \varrho V^{2}$

is the total energy, $\varrho=\varrho_{\mathrm{e}}+\varrho_{1}$ is the mass density, $V=$ $V_{\mathrm{e}}+V_{1}$ is the flow velocity, $p=p_{\mathrm{e}}+p_{1}$ is the pressure, $\gamma$ is the adiabatic index, and $(, t)$ and $(, x)$ denote partial derivatives in time and space, respectively. The subscript "e" refers to equilibrium or "background" values and the subscript "1" refers to wave fluctuations.

The source terms $S_{\rho}$ and $S_{V}$ represent mass and momentum sources, respectively. We note that these sources are merely 
phenomenological, and that mass, momentum and energy is not conserved due to these sources. The term $S_{\rho}$ has the meaning of a temporal derivative of a random mass density and $S_{V}$ generates a wave field. These terms are described below for the case of a static equilibrium.

The equilibrium state which is set initially at $t=0$ is defined with a varying background density,

$\varrho_{\mathrm{e}}(x, t)=\varrho_{0}+\varrho_{\mathrm{r}}(x, t)$,

accompanied with a constant pressure $p_{\mathrm{e}}=p_{0}$. In equilibrium, $S_{V}=0$, and with constant pressure, the velocity remains at zero such that $V_{\mathrm{e}}=0$. This definition of the equilibrium state leads to the relation

$\varrho_{\mathrm{e}, t}=\varrho_{\mathrm{r}, t}=S_{\varrho}$

since $\varrho_{0}$ is constant.

The random part of the background mass density, $\varrho_{\mathrm{r}}$, is chosen to be statistically homogeneous (i.e., the statistical properties are independent of the coordinates in space and time), with Gaussian power spectrum

$E(k, \omega)=\frac{\sigma^{2} l_{\mathrm{x}} l_{\mathrm{t}}}{\pi^{2}} \mathrm{e}^{-k^{2} l_{\mathrm{x}}^{2}} \mathrm{e}^{-\omega^{2} l_{\mathrm{t}}^{2}}$

Here, $\sigma^{2}$ is the variance of the normalized fluctuations $\varepsilon=$ $\varrho_{\mathrm{r}} / \varrho_{0}$ and $l_{x}$ and $l_{t}$ are the correlation length and the correlation time, respectively.

\subsection{The associated wave equation}

Linear theory shows that a coherent field exists only when the source in the wave equation correlates to the coefficients in the wave equation (Skartlien 2002). We linearize the mass, momentum and energy equations and obtain to leading order the perturbed wave equation

$L_{0}[V]+L_{1}[V]=S$,

$L_{0}[V] \equiv V_{, t t}-c_{0}^{2} V_{, x x}$,

$L_{1}[V] \equiv \varepsilon V_{, t t}+\varepsilon_{, t} V_{, t}$,

$\varepsilon \equiv \frac{\varrho_{\mathrm{r}}}{\varrho_{0}}$

where $c_{0}=\sqrt{\gamma p_{0} / \varrho_{0}}$ is the unperturbed sound speed. The model generates a wave field $V(x, t>0)$ which is described accurately by this wave equation when the wave amplitude is kept sufficiently small. The unperturbed wave operator is $L_{0}$, involving the constant sound speed $c_{0}$. The perturbed part of the wave operator is $L_{1}$. The term $\varepsilon_{, t} V_{t}$ is a random source/sink, and the term $\varepsilon V_{, t t}$ can be linked to random fluctuations in propagation speed. The wave source is

$S=\left[S_{V}(1+\varepsilon)\right]_{, t}-(\gamma-1)\left[(1+\varepsilon) V S_{V}\right]_{, x}$.

The term $(\gamma-1)\left[(1+\varepsilon) V S_{V}\right]_{, x}$ is smaller than the first one such that to leading order, $S=\left[S_{V}(1+\varepsilon)\right]_{, t}$. Only the leading order expression will be considered in the following. The source $S_{\varrho}$ enters through $\varrho_{\mathrm{r}}$ in $\varepsilon$. The linearized momentum equation, Eq. (2), relates the source term $S_{V}$ to the perturbed quantities:

$S_{V}=V_{, t}+\frac{1}{\varrho_{\mathrm{e}}} p_{, x}$

The existence of the coherent field requires a correlation between the coefficients in the perturbed operator $L_{1}\left(\varepsilon\right.$ or $\left.\varepsilon_{, t}\right)$ and the source $S$. For these correlation functions, we find

$\langle\varepsilon S\rangle=\left\langle\varepsilon\left[S_{V}\right]_{, t}\right\rangle+\left\langle\varepsilon\left[S_{V} \varepsilon\right]_{, t}\right\rangle$

and

$\left\langle\varepsilon_{, t} S\right\rangle=\left\langle\varepsilon_{, t}\left[S_{V}\right]_{, t}\right\rangle+\left\langle\varepsilon_{, t}\left[S_{V} \varepsilon\right]_{, t}\right\rangle$,

where the brackets \langle\rangle denote ensemble averaging ${ }^{1}$. The correlation function can then provide a coherent field, if $\varrho_{\mathrm{r}}$ and $S_{V}$ (or their derivatives) are correlated.

\subsection{Random wave source}

For the correlated case, we use a spatially localized source which is proportional to the density fluctuations,

$S_{V}(x, t)=S_{0} \varepsilon(x, t) \exp \left[-\left(x-x_{0}\right)^{2}\right], \quad S_{0}=$ const.

The correlation function between the source and the normalized density fluctuations is now

$\left\langle S_{V}(x, t) \varepsilon\left(x^{\prime}, t^{\prime}\right)\right\rangle=S_{0}\left\langle\varepsilon(x, t) \varepsilon\left(x^{\prime}, t^{\prime}\right)\right\rangle \exp \left[-\left(x-x_{0}\right)^{2}\right]$.

For the uncorrelated case, we modulate the correlated source above with a spatially random function $S_{x}(x)$,

$S_{V}(x, t)=S_{x}(x) \varepsilon(x, t) \exp \left[-\left(x-x_{0}\right)^{2}\right]$.

The correlation function vanishes,

$\left\langle S_{V}(x, t) \varepsilon\left(x^{\prime}, t^{\prime}\right)\right\rangle=$

$\left\langle S_{x}(x)\right\rangle\left\langle\varepsilon(x, t) \varepsilon\left(x^{\prime}, t^{\prime}\right)\right\rangle \exp \left[-\left(x-x_{0}\right)^{2}\right]=0$,

since the ensemble average $\left\langle S_{x}(x)\right\rangle=0$.

\section{Ensemble averaging and the coherent field}

There are at least two ways of observing independent realizations of the wave field such that ensemble averaging can be performed. 1) One can observe one single field of view within $N$ time windows that are separated by more than one correlation time. The medium needs to be statistically stationary in time, but not necessarily statistically homogeneous in space. 2) One can observe $N$ different fields of view (with sufficient spatial separation) within the same time window. The medium needs to be statistically homogeneous in space, but not necessarily statistically stationary in time. One can also imagine a combination of 1) and 2).

1 The correlation functions depend on $x$ and $t:\langle A B\rangle=$ $\left\langle A(x, t) B\left(x^{\prime}, t^{\prime}\right)\right\rangle$. For statistically homogeneous conditions, the correlation function depends only on coordinate differences, such that $\langle A B\rangle=C\left(t-t^{\prime}, x-x^{\prime}\right)$. 
After having observed $N$ realizations of the wave field, we perform ensemble averaging,

$\langle V(x, t)\rangle_{N}=\frac{1}{N} \sum_{j=1}^{N} V_{j}(x, t)$.

Is is clear that as $N \rightarrow \infty$, the ensemble average converges to the coherent field $\langle V\rangle_{\infty}$. In the uncorrelated case, the ensemble average converges to zero.

In order to evaluate the global convergence, we calculate the mean energy of the ensemble averaged field,

$e_{N}=\overline{\langle V(x, t)\rangle_{N}^{2}}$,

where the bar denotes averaging over the $(x, t)$-plane. When separating the field in coherent and incoherent parts, $V=$ $\langle V\rangle_{\infty}+\delta V$, one can show that the expectation value of the mean energy is (omitting the $(x, t)$ arguments),

$E\left[e_{N}\right]=\overline{\langle V\rangle_{\infty}^{2}}+\overline{\frac{E\left[(\delta V)^{2}\right]}{N}}$

The first term is the mean energy of the coherent field, and the second term is the mean energy of the incoherent field, divided by the number of realizations we have averaged over. Thus, for the uncorrelated case,

$e_{N}($ uncorrelated $)=O\left(\overline{E\left[(\delta V)^{2}\right]}\right) / N$

and for the correlated case,

$e_{N}($ correlated $)=O\left(\overline{\langle V\rangle_{\infty}^{2}}\right)$.

\section{Numerical results}

Our intention here is to verify numerically the non-existence (existence) of the coherent field when the density fluctuations $\varrho_{\text {r }}$ are uncorrelated (correlated) to the source $S_{V}$, and to compare the frequency shifts of the line profiles in the ensemble averaged power spectra. The standard deviation of the relative density fluctuation $\varepsilon$ is chosen as $\sigma=0.05$, corresponding to the weak scattering regime. The amplitude of the source is set such that we remain in the linear regime where $\left|V / c_{0}\right| \ll 1$.

To perform numerical simulations, we have adapted the code CLAWPACK (LeVeque 2002), a packet of Fortran routines for solving hyperbolic equations (e.g., Murawski 2002). We use a homogeneous grid in space $x$ and time $t$, and periodic boundaries in $x$ for simple adaption to spectral analysis via the Fast Fourier Transform.

\subsection{Coherent field}

Figure 1 shows the mean energy of the ensemble averaged field, $e_{N}=\overline{\langle V(x, t)\rangle_{N}^{2}}$, in both correlated and uncorrelated cases. When the density fluctuations are correlated to the source, the mean energy converges to the mean energy of the coherent field, $\overline{\langle V\rangle_{\infty}^{2}}$. When the density fluctuations are not correlated to the source, the mean energy converges to zero $\left(e_{N}=\right.$ $O\left(\overline{E\left[(\delta V)^{2}\right]}\right) / N$ ) because the coherent field is zero. We note that in the uncorrelated case, the field is purely incoherent, while in the correlated case, it has both coherent and incoherent contributions.

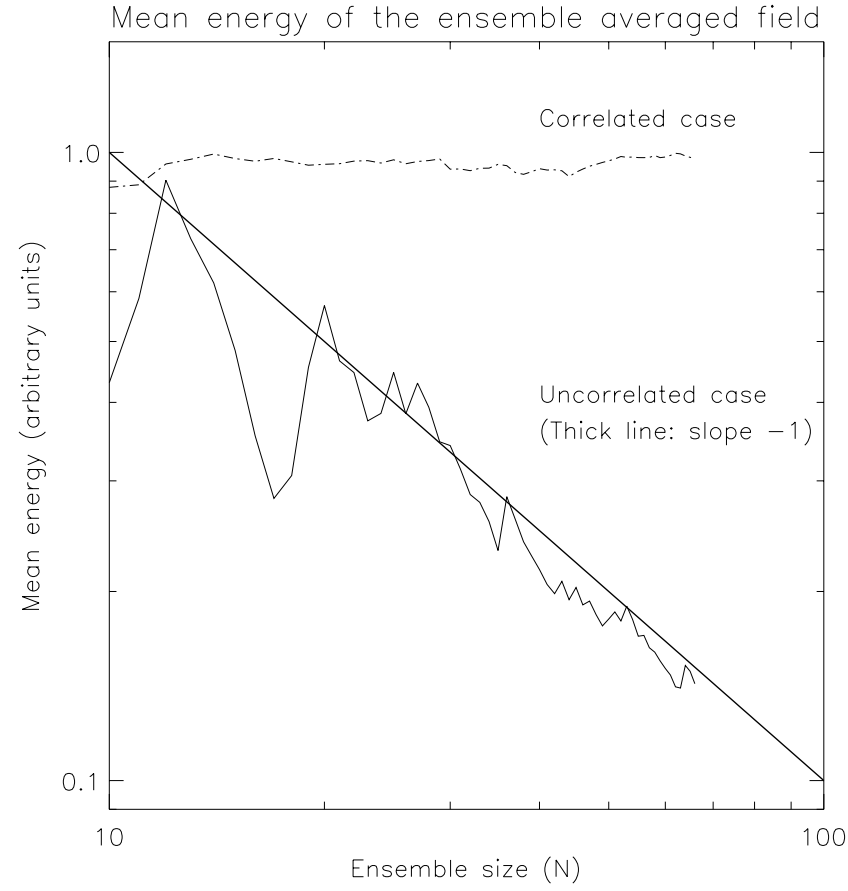

Fig. 1. Convergence of the mean energy of the ensemble averaged fields. The mean energy converges to the mean energy of the coherent field in the correlated case (dashed line). For the uncorrelated case, the mean energy converges to zero because the coherent field is zero in this case (full line). The mean energy is proportional to $1 / N$ in the uncorrelated case, and results in a slope of -1 in the given log-log plot (thick line).

\subsection{Power spectra}

Figure 2 shows ensemble averaged power spectra of the velocity field as function of frequency $\omega$ in the correlated case (with the wavenumber $k$ as parameter). The power maxima in the frequency spectra are close to the frequencies dictated by the unperturbed dispersion relation $\omega_{0}=c_{0} k$. Thus, we get only one power maximum for each wavenumber, and most of the energy is concentrated around these frequencies. Previous studies on similar problems show that stochastic perturbations in the medium can lead to shifts in frequency for the power maxima, or for the line profile as a whole (e.g., Murawski et al. 2001 (undriven free waves) or Skartlien 2002 (stochastically driven waves, uncorrelated medium and source)).

Figure 3 shows (for both cases) the relative frequency shifts $\left(\omega-c_{0} k\right) /\left(c_{0} k\right)$ of the line profiles for different $k$. We see that a correlation between the source and the density fluctuations is also manifested in the frequency shifts. The shifts are mostly positive in the uncorrelated case (squares). In the correlated case, the shifts are much smaller and mostly negative (crosses). These differences demonstrate the importance of accounting for a stochastic wave source when attempting to explain p-mode and f-mode spectra.

We note that the expectation value of the power spectrum $E[P(k, \omega)]$ can be split in coherent and incoherent parts,

$E[P]=\left\langle\tilde{V} \tilde{V}^{*}\right\rangle_{\infty}=\langle\tilde{V}\rangle_{\infty}\langle\tilde{V}\rangle_{\infty}^{*}+\left\langle\delta \tilde{V} \delta \tilde{V}^{*}\right\rangle_{\infty}$, 

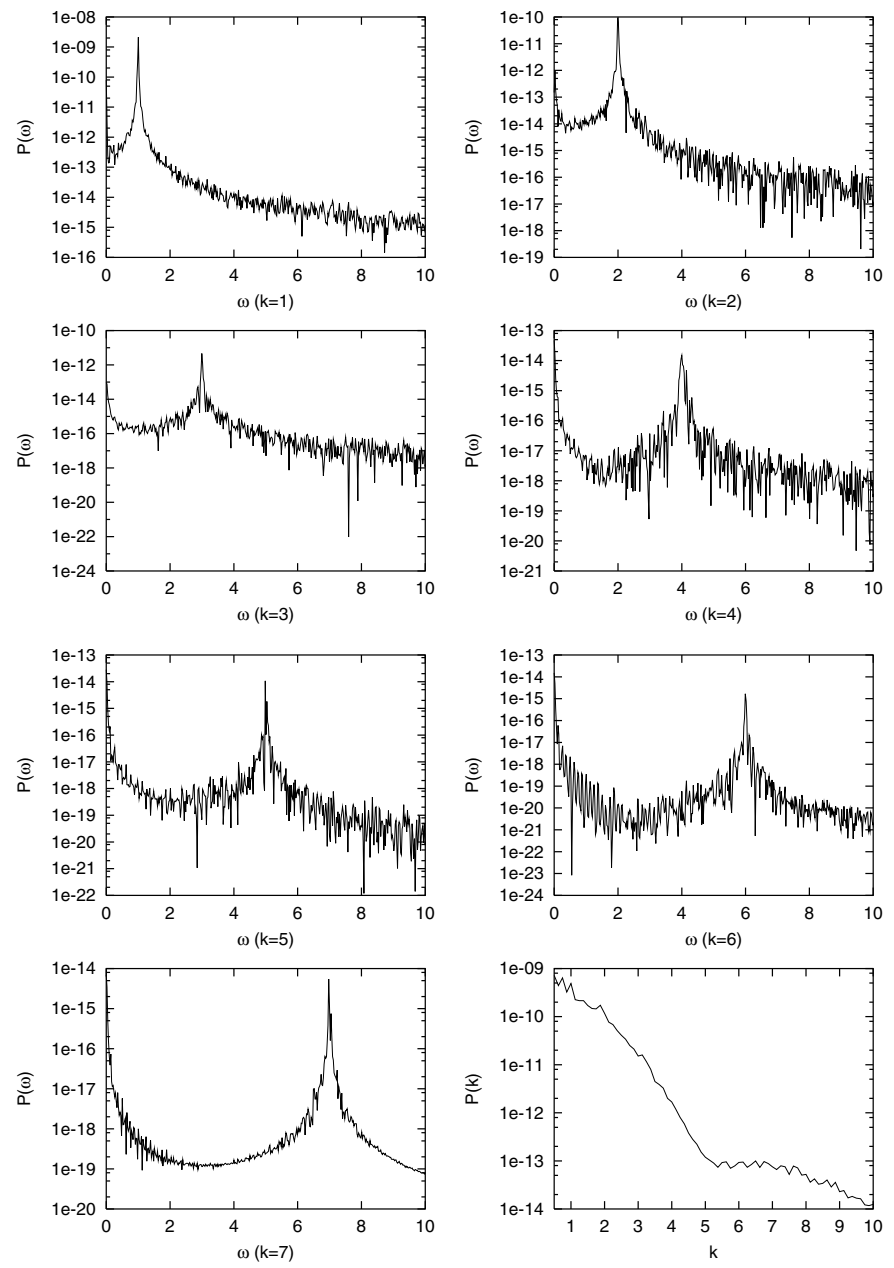

Fig. 2. Ensemble averaged power spectra for the correlated case as function of frequency $\omega$ for different wavenumbers. The power maxima in the frequency spectra are close to the frequencies dictated by the unperturbed dispersion relation $\omega_{0}=c_{0} k$. The bottom right panel shows the power spectrum of the ensemble averaged field (sampled at a specific time), showing a smooth distribution of energy over wavenumber.

by splitting the wavenumber-frequency Fourier transform of $V$ in coherent and incoherent parts, $\tilde{V}=\langle\tilde{V}\rangle_{\infty}+\delta \tilde{V}$. The contribution $\left\langle\delta \tilde{V} \delta \tilde{V}^{*}\right\rangle_{\infty}$ is the incoherent power, and the contribution $\langle\tilde{V}\rangle_{\infty}\langle\tilde{V}\rangle_{\infty}^{*}$ is the coherent power. It is important to note that the mean field dispersion relation for the coherent field can only predict frequencies of maximum coherent power, and not frequencies of maximum total power. In the correlated case, we have both coherent and incoherent power, but in the uncorrelated case we have only incoherent power. Thus, the mean field dispersion relation is in the worst case (uncorrelated case) irrelevant in the context of the power spectrum. In order to explain the different frequency shifts in the two cases, one can expand the power spectrum in a series as in Skartlien (2002).

\section{Summary and discussion}

We have studied stochastic sound waves in a one-dimensional random medium by numerical solution of the Euler equations.

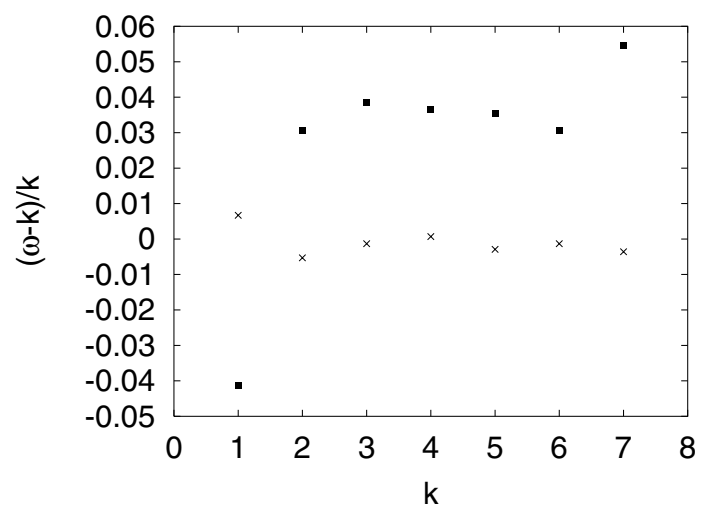

Fig. 3. Relative frequency shifts for the correlated case (crosses) and for the uncorrelated case (squares). The frequency shifts are mostly positive in the uncorrelated case. In the correlated case, the shifts are much smaller and mostly negative.

We considered two cases in which the background density and wave source were either correlated or uncorrelated. The coherent field is non-zero in the correlated case, yielding both coherent and incoherent fields. In the uncorrelated case, there is only an incoherent field.

The physical content of this result is that there must be a correlation between the perturbed wave operator and the wave source, for the coherent field to exist. The perturbed wave operator corresponds to fluctuations in the propagation characteristics of the waves, and in our case it was the background density fluctuations which provided these fluctuations. It is also important to note the diagnostic potential; we can in principle determine from observations whether or not there is a correlation between the wave source and the physical quantity which provides the fluctuating propagation characteristics.

Much attention has been given to frequency shifts in the solar p-mode and f-mode spectra in the past, motivated by the desire to understand the solar interior. An important question is therefore: do we make significant errors in predicted frequency shifts using deterministic helioseismic modelling? To answer this question we need good stochastic models. The usual approach in stochastic modelling so far is to use mean-field dispersion relations to arrive at solar eigenfrequencies. Is this a valid approach? The mean-field dispersion relation governs the coherent field only, and one can therefore only calculate eigenfrequencies for the coherent field. There are two strong reasons for questioning the use of mean-field dispersion relations: 1) the coherent field can simply be non-existent, as we have demonstrated for the uncorrelated case, and 2) the coherent field might only contribute with a small amount of power to the power spectrum.

We demonstrated that the frequency shifts in the power spectrum depend on the correlation between the stochastic source and the background fluctuations. An explanation of this will be attempted in a forthcoming paper by using linear theory to calculate the expectation value of the power spectrum without going through dispersion relations (Skartlien 2002). Much work still needs to be done in this direction, and simple 
homogeneous models are going to give us more valuable insight. We encourage researchers in the field of helioseismology to determine the magnitude of the errors made in both deterministic modelling and in stochastic modelling using meanfield dispersion relations.

Acknowledgements. M.S.'s and K.M.'s works were financially supported by the State Committee for Scientific Research in Poland, KBN grant no. 2 PO3D 016 25. R.S. thanks the co-authors and Tore Smestad.

\section{References}

Gough, D. O. 1994, in Astrophysical Fluid Dynamics, ed. J.-P. Zahn, \& J. Zinn-Justin (Amsterdam: Elsevier)

LeVeque, R. 2002, Finite volume methods for hyperbolic problems (Cambridge: Cambridge Univ. Press)

Murawski, K. 2002, Analytical and numerical methods for wave propagation in fluid media (Singapore: World Scientific)

Murawski, K., Nocera, L., \& Mędrek, M. 2001, A\&A, 376, 708

Nocera, L., Medręk, M., \& Murawski, K. 2001, A\&A, 373, 303

Skartlien, R. 2002, ApJ, 578, 621 